\title{
DESARROLLO E INSTRUMENTACIÓN DE UN VELERO AUTÓNOMO DE 2 METROS DE ESLORA PARA LA RECOGIDA DE DATOS OCEANOGRÁFICOS
}

\author{
Esteban Chacón Mosquera \\ Universitat Politècnica de Catalunya (UPC), Jordi Girona 31, 08034 Barcelona, estebanfex@gmail.com \\ Carles Manich Vallès \\ Facultat de Nàutica de Barcelona (UPC), Pla de Palau 18, 08003 Barcelona, carles.manich@gmail.com \\ Joan Antoni Sastre Caballol \\ Facultat de Nàutica de Barcelona (UPC), Pla de Palau 18, 08003 Barcelona, crasty9715@gmail.com \\ Josep Eudald Mesegué Basallo \\ Facultat de Nàutica de Barcelona (UPC), Pla de Palau 18, 08003 Barcelona, eudald.mesegue@gmail.com \\ Rosa M. Fernández-Cantí \\ Facultat de Nàutica de Barcelona (UPC), Pla de Palau 18, 08003 Barcelona, rosa.mari.fernandez@upc.edu
}

\begin{abstract}
Resumen
Esta contribución presenta la construcción y automatización de un velero de 2 metros de eslora concebido para navegar de manera autónoma y llevar a cabo mediciones cientificas. Se describe la instrumentación y la recogida de información por parte de los distintos sensores. La transmisión de esta información se realiza en forma de trama al procesador principal, el cual ejecuta un algoritmo de control para generar las órdenes a los servos de los timones y al servo del alerón de la vela. El procesador principal también es el encargado de la presentación de los datos en el formato JavaScript Object Notation (JSON). Se comentan aspectos concretos de la maniobra de la vela y se presenta el resultado experimental de las primeras pruebas de mar.
\end{abstract}

Palabras clave: Navegación autónoma, velero de 2 metros de eslora, Raspberry Pi, instrumentación electrónica.

\section{INTRODUCCIÓN}

Dentro del ámbito de la navegación autónoma, el desarrollo de embarcaciones de pequeña eslora (drones marinos), capaces de operar con total autonomía y transmitir o almacenar los datos, es uno de los campos que más interés está recibiendo por parte de los investigadores [5], [6].

El uso de este tipo de embarcaciones en estudios científicos, como por ejemplo los asociados a aspectos concretos del cambio climático y/o la biodiversidad, es muy interesante por diversos motivos, destacando entre ellos la seguridad de las personas, el coste económico y la versatilidad que ofrecen este tipo de embarcaciones frente a las tradicionales expediciones en buques oceanográficos [7]. La Figura 1 muestra el velero autónomo Sensailor, objeto de este trabajo.

El desarrollo de las comunicaciones vía satélite y el llamado internet de las cosas permite recibir a distancia información sobre la posición de los artefactos marinos y la lectura de sus sensores (ver, por ejemplo, [2]), mientras que las mejoras en el propio diseño de las embarcaciones, su instrumentación y algoritmos de navegación permiten que estas puedan operar de manera autónoma a más distancia, durante más tiempo y en un rango más amplio de condiciones de navegación, [1], [3].

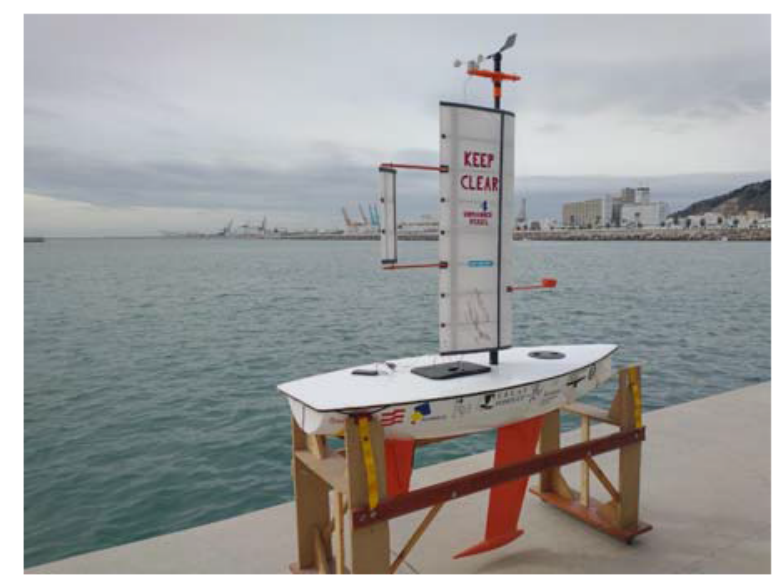

Figura 1: Velero autónomo Sensailor 
El diseño y construcción de este tipo de artefactos marinos es multidisciplinar puesto que incluye áreas tan diversas como la construcción naval, los métodos de propulsión, la instrumentación electrónica, las comunicaciones y la automatización.

Con respecto a la construcción naval, dentro del campo de los drones marinos de superficie podemos encontrar diversos tipos de embarcaciones y diversas configuraciones. Los veleros son un caso particular cuyo sistema de propulsión primario es la fuerza del viento. Los veleros autónomos presentan la ventaja de que la propulsión no consume energía con lo que los medios de captación (p. ej., unas placas solares) y las baterías pueden destinarse exclusivamente a alimentar la instrumentación (sensores de navegación y científicos, actuadores sobre timones y vela).

Con respecto a la instrumentación, la aparición en la última década de tecnologías de bajo coste, prototipado rápido y código libre ha incrementado considerablemente el número de proyectos en este ámbito y ha propiciado la aparición de regatas donde los investigadores pueden optimizar sus diseños e ir mejorando continuamente el comportamiento y las prestaciones de sus embarcaciones [8]. Es el caso de la Microtransat (https://www.microtransat.org/) y el World Robotic Sailing Championship (https://www.roboticsailing.org/), por citar solo dos de las más conocidas donde pueden participar veleros autónomos de pequeña eslora.

El resto del documento se organiza como sigue: En la Sección 2 se presenta el velero Sensailor y se describen brevemente sus características constructivas y de maniobra. A continuación, en la Sección 3 se describe la instrumentación (sensores y actuadores) y se explica cómo se realiza la transmisión de datos. En la Sección 4 se resumen los resultados experimentales, se discuten algunos aspectos del diseño y se proponen mejoras. Finalmente, en la Sección 5 se extraen las principales conclusiones del trabajo.

\section{VELERO AUTÓNOMO SENSAILOR}

El velero Sensailor [1], [7] (ver Figura 1) es una embarcación de 2 metros de eslora, con sistema de navegación automática y autosuficiencia energética. Está diseñado para navegar en la zona 2 (hasta 60 millas náuticas de la costa) y soportar condiciones meteorológicas de categoría 6 en la escala de Beaufort. La distribución de masas y forma del buque permite recuperar la verticalidad en caso de vuelco. El casco es estanco a fin de proteger la instrumentación interna y el conjunto es fácilmente desmontable y transportable.

\subsection{CONSTRUCCIÓN}

El detalle de la construcción incluyendo los cálculos y simulaciones de estabilidad, se puede consultar en [7].

\subsubsection{Casco}

Para la construcción del casco se ha creado un negativo del molde con espuma de poliuretano, y de este se ha extraído un molde, a partir del cual se ha laminado el casco. Para el laminado se ha usado resina de poliéster y fibra de vidrio. Antes de pintar se ha aplicado imprimación.
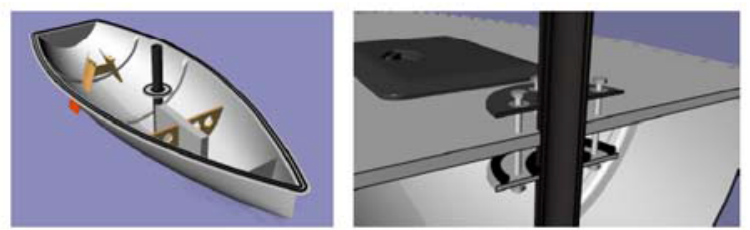

Figura 2: Diseño del casco. Mástil [7]

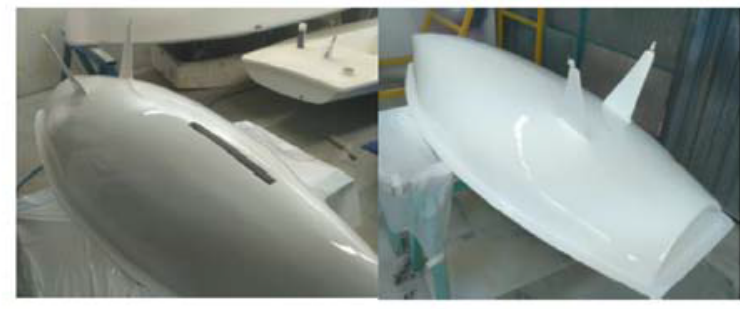

Figura 3: Construcción del casco [7]

\subsubsection{Apéndices}

Las Figuras 4 a 6 muestran el diseño de los apéndices. El velero cuenta con dos timones que se mueven a la vez y en el mismo sentido, accionados cada uno por un servomotor independiente. Este diseño añade redundancia al sistema y puede ser útil en situaciones de colisión o arrastre de objetos indeseados tales como plásticos, redes, etc.

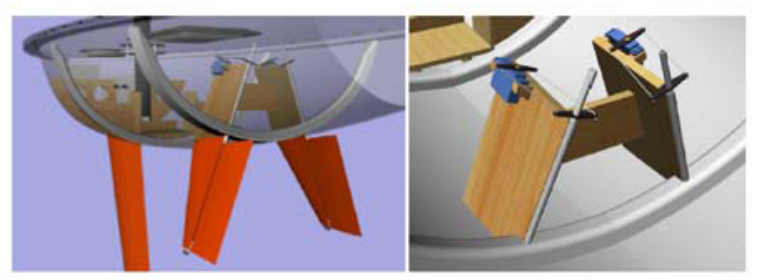

Figura 4: Timones [7]

Por su lado, el bulbo de la orza es un lastre de plomo de $10 \mathrm{~kg}$. 


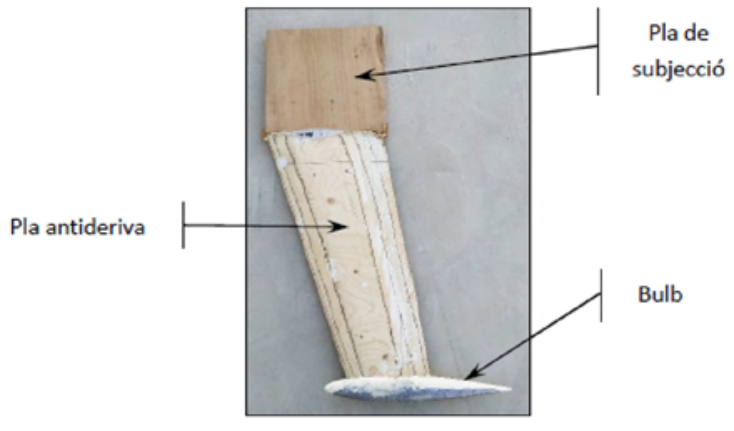

Figura 5: Construcción de la orza [7]

Finalmente, el mástil de la vela sirve también como soporte del anemómetro, veleta y luz de posición, y en su interior se encuentran los cables que conectan estos dispositivos con el interior del casco.

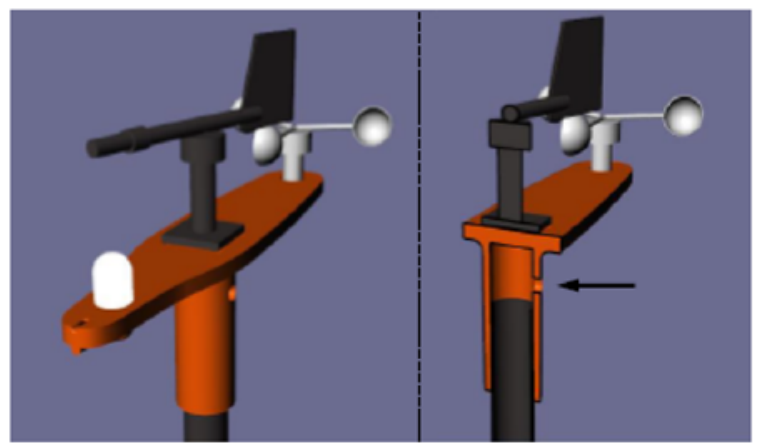

Figura 6: Instrumentación en el mástil [7]

\subsubsection{Propulsión: vela y alerón}

El sistema propulsivo consta de una vela rígida en cuyo interior se encuentra el servo que gobierna el ángulo del alerón por medio de una varilla.

El par de fuerzas generadas por el alerón y la vela logra orientar a esta última con el ángulo de ataque (ángulo respecto al viento) deseado. Este sistema permite ahorrar un elemento que mueva y fije la posición de la vela, reduciendo notablemente el consumo.

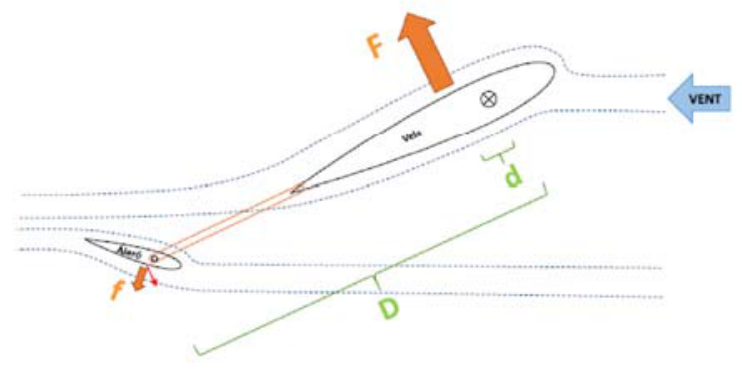

Figura 7: Par de fuerzas de la vela y el alerón
Tanto la vela como el alerón tienen una sección transversal invariable, basada en un perfil aerodinámico de la base de datos NACA, en concreto un perfil simétrico, el NACA 0015.

Dicho perfil, seleccionado para satisfacer las especificaciones de navegabilidad [7], al recibir viento, genera una fuerza que, después de los correspondientes cambios de los ejes de referencia, se puede descomponer en dos (ver Figura 8): la fuerza que contribuye al avance de la embarcación $\left(F^{\mathrm{L}}\right)$, y la que afecta a su escora $\left(F^{t}\right)$. Con la variación del ángulo de ataque de la vela, las fuerzas obtenidas varían.

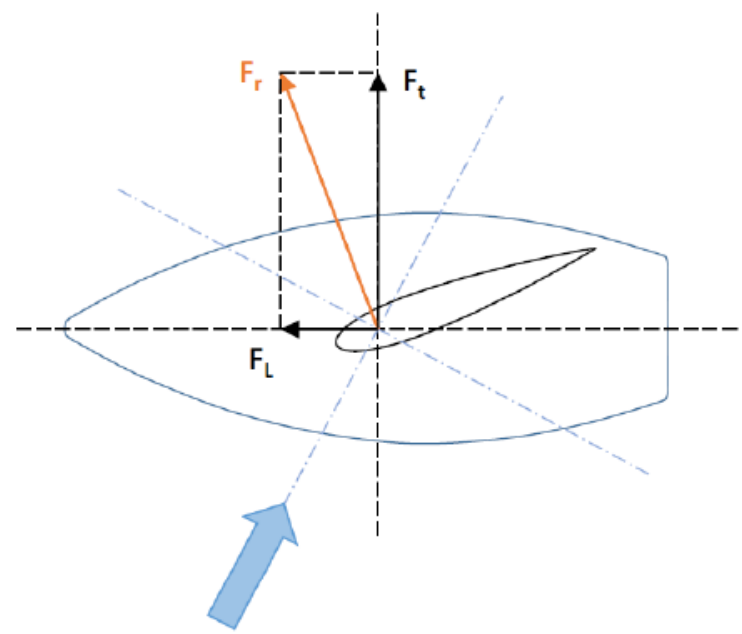

Figura 8: Fuerza resultante de la vela respecto a la embarcación

La fuerza propulsiva $F^{\mathrm{L}}$ se opone a la fuerza de resistencia al avance del casco. Cuando la resistencia es superada, la embarcación avanza.

Para dimensionar la superficie vélica, debe tenerse en cuenta el concepto de velocidad de diseño. Esta velocidad $V_{\max }$ es la máxima a la que podrá navegar la embarcación, por las características del casco. Para calcular dicha velocidad, se puede usar:

$$
V_{\max }=2.4 \sqrt{L}
$$

donde $L$ es la eslora del barco, en este caso $2 \mathrm{~m}$. Así pues, la velocidad máxima de diseño es de $3.34 \mathrm{kn}$, y se usa este parámetro para calcular el dimensionamiento de la vela, de forma que genere la fuerza necesaria para navegar a esta velocidad.

\subsection{MANIOBRA}

Las condiciones idóneas para el funcionamiento y en las cuales se garantiza su seguridad e integridad están comprendidas entre los 5 y los 20 nudos de viento. 
En el primer prototipo, existe una limitación en el giro de la vela, que no puede ser de $360^{\circ}$. Esto se debe a que el cable que alimenta y controla el servo del alerón, que se encuentra en el interior del mástil, podría enrollarse indefinidamente con los giros de la vela. Esta limitación añade complejidad a la navegación, obligando a usar maniobras de acuerdo a ésta, para que el velero reciba el viento necesario para avanzar.

El algoritmo de control tiene por tanto en cuenta tanto la dirección del viento como el rumbo hacia las coordenadas deseadas (waypoint, WP), así como el límite de giro de la vela. En función de todas estas entradas, actúa sobre los servos de los timones y alerón de vela.

La Figura 9(b) muestra cuál debe ser la posición de la vela en función del rumbo hacia el waypoint deseado y la dirección del viento. Debido a que la vela no puede girar libremente $360^{\circ}$, la virada siempre es por proa.

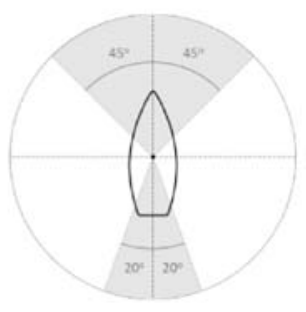

(a)

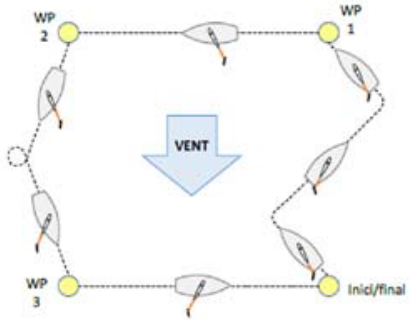

(b)
Figura 9: Maniobra [7]

Los grados indicados en la Figura 9(a) representan los ángulos del viento respecto a la embarcación para los que se puede lograr avance.

\section{INSTRUMENTACIÓN}

Para poder navegar de forma totalmente autónoma, el velero incluye tres dispositivos con capacidad de procesado. Una placa Arduino UNO, una Raspberry Pi 4 y una ESP32. La Figura 10 muestra a cuál de los tres procesadores van conectados los diferentes sensores y actuadores.

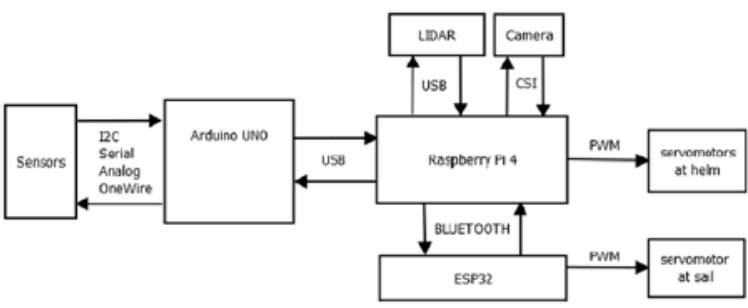

Figura 10: Procesadores, sensores y actuadores

\subsection{PROCESADORES}

El Arduino UNO es el encargado de recoger la información de los sensores que operan a $5 \mathrm{~V}$. Estos incluyen los sensores más relevantes para la navegación autónoma (GPS, anemómetro, veleta, magnetómetro), para la recogida de datos (temperatura, rayos UV) y para decidir cuándo encender la luz nocturna de posición (sensor LDR).

La función de la placa Arduino es recoger todos los datos y transmitirlos a la Raspberry Pi por medio de una conexión USB. También es la encargada de encender la luz de posición cuando la luminosidad detectada por el sensor LDR se sitúa por debajo de un umbral.

La Raspberry Pi a su vez recibe la información de una cámara NoIR que no tiene filtro de luz infrarroja (para visión nocturna) y un sensor LIDAR (para la detección de obstáculos) cuya información también influirá en las señales de mando que operan sobre los servos de los dos timones y alerón de la vela.

La Figura 11 muestra las placas Raspberry y Arduino. Estas van montadas en una caja hermética dentro del casco.

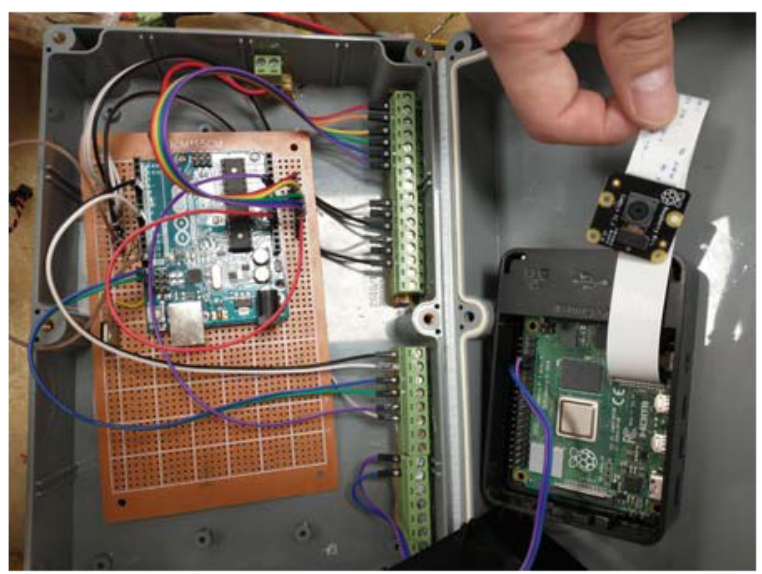

Figura 11: Placas Arduino y Raspberry

La placa Raspberry dispone también de una tarjeta de memoria SD de 32GB antichoque, resistente a bajas y altas temperaturas, impermeable y blindada frente a rayos $\mathrm{X}$.

La información de los diferentes dispositivos se almacena en dos ficheros de texto, uno para la información de navegación y otro para la información científica recogida. El formato escogido para almacenar y visualizar los datos es JavaScript Object Notation (JSON), adicionalmente a los datos se les incluye fecha y hora para un registro más completo. 
En la Raspberry se ha programado, con ayuda del lenguaje Python, un algoritmo de inteligencia artificial basado en aprendizaje por refuerzo que es el encargado de generar las señales de mando hacia los servos de los dos timones y el servo del alerón, a partir de la información de los sensores.

En el caso del servo del alerón, y para evitar la posible torsión de los cables dentro del mástil de la vela, se ha optado por que la Raspberry 4 transmita la señal de control por Bluetooth hacia el procesador ESP32 situado en la vela (ver Figura 15).

\subsection{SENSORES Y ACTUADORES}

\subsubsection{Sensores}

Los dispositivos sensores de $5 \mathrm{~V}$ relacionados directamente con la navegación conectados al Arduino son los siguientes:

Un sensor GPS, modelo RCmall FZ2922 transmite los datos en standard NMEA y se comunica con el Arduino mediante una conexión serie. Para ello se han configurado los pines D5 y D8 como $\mathrm{Rx} / \mathrm{Tx}$. Este módulo devuelve la posición GPS en tiempo real cada segundo, así como la distancia y rumbo al waypoint.

Un magnetómetro, modelo MPU-9250, conectado al puerto I2C (SDA/SDL) del Arduino permite conocer el rumbo del Sensailor para posteriormente compararlo con la información del GPS. Este dispositivo lleva incorporado un acelerómetro, que permitirá, en un futuro monitorizar y controlar la escora de la embarcación.

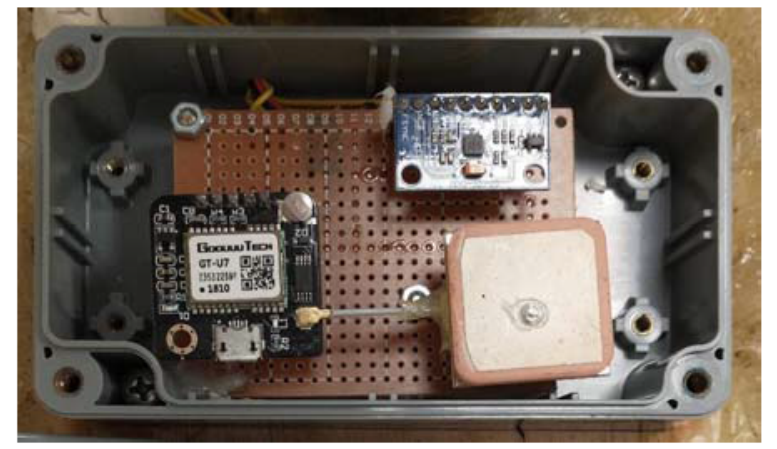

Figura 12: Detalle del GPS, su antena y el magnetómetro

Un anemómetro, conectado a través de un diodo al pin D2. La salida del anemómetro es un tren de pulsos cuya frecuencia es proporcional a la velocidad del viento. A fin de evitar el efecto de rebote se ha decidido detectar los pulsos por flanco ascendente.
Una veleta HYXC-FX alimentada a $12 \mathrm{~V}$ para medir la dirección del viento, cuya salida de $5 \mathrm{~V}$ se conecta al pin A0 del Arduino.

Aparte de estos sensores, la placa Arduino también recibe señal de los siguientes dispositivos:

Un sensor de temperatura, modelo DS18b20, onewire, conectado a la placa a través de una resistencia de $4 \mathrm{k} \Omega$.

Un sensor LDR simple, conectado mediante un divisor de tensión al pin A1, calibrado de manera que cuando la tensión que mide la luminosidad baje de $700 \mathrm{mV}$ se encienda la luz de posición.

Un sensor de rayos UV modelo ML8511 conectado al pin A2.

Finalmente, el velero dispone de dos sensores más para la detección de obstáculos: un LIDAR modelo RPLIDAR A1 y una cámara nocturna modelo RPi NoIR Camera V2. Esta última se muestra en la Figura 11. Ambos sensores envían la señal a la placa Raspberry Pi para el posterior procesamiento de datos.

\subsubsection{Transmisión y presentación de datos}

Para el intercambio de información entre el Arduino (encargado de recibir los datos de los sensores de 5V) y la Raspberry Pi (encargada de generar las señales de control) se ha diseñado una trama específica que se transmite cada segundo por medio de la conexión USB entre las dos placas.

Tabla 1: Campos de la trama de sensores.

\begin{tabular}{|c|l|}
\hline Id & Dispositivo \\
\hline A & Veleta \\
B & Anemómetro \\
C & Magnetómetros \\
D & GPS velocidad \\
E & GPS latitud \\
F & GPS longitud \\
G & Sensor UV \\
H & Sensor LDR \\
I & Sensor DS18b20 temperatura \\
J & Rumbo a destino \\
K & Distancia a destino \\
\hline
\end{tabular}

Se trata de una transmisión por multiplexación en el tiempo síncrona de manera que cada dispositivo tiene asignado un slot de tiempo fijo de duración $50 \mathrm{~ms}$. Puesto que la frecuencia de adquisición de datos de cada sensor es diferente, los campos se actualizan solo cuando hay nuevos datos $\mathrm{y}$, mientras estos no se actualizan, mantienen la lectura anterior. La Tabla 1 muestra el campo asignado a cada uno de los sensores instalados actualmente en el velero. 
La Figura 13 muestra el diseño de la trama. Notar que se ha dejado margen para la inclusión de nuevos dispositivos.

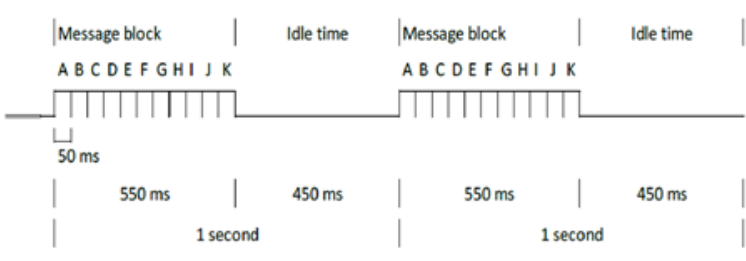

Figura 13: Configuración de trama

\subsubsection{Presentación de datos y algoritmo de control}

Las medidas de los diferentes sensores se almacenan en dos ficheros de texto (uno para navegación y otro para datos oceanográficos) y se presentan en formato JSON.

Por otro lado, el algoritmo de control actualmente implementado es un algoritmo de aprendizaje no supervisado por refuerzo basado en una red neuronal de 2 capas que contienen 64 neuronas densamente interconectadas el cual utiliza como entradas la dirección y posición del Sensailor así como del viento, y como salidas el giro de los servomotores, utilizando como criterio de recompensa la velocidad del bote, la disminución de la distancia a las coordenadas de destino (waypoint) y el rumbo correcto hacia dicho waypoint. Los detalles pueden consultarse en [1].

\subsubsection{Actuadores}

La navegación se controla por medio de tres servomotores: uno para cada timón y uno para el alerón de la vela.

Aunque cada timón tiene su servo, la orden a los dos servos es la misma. Se ha escogido el modelo RCTecnic-S0150 de $15 \mathrm{~kg} \cdot \mathrm{cm}$ alimentados a $6 \mathrm{~V}$ (ver Figura 14).
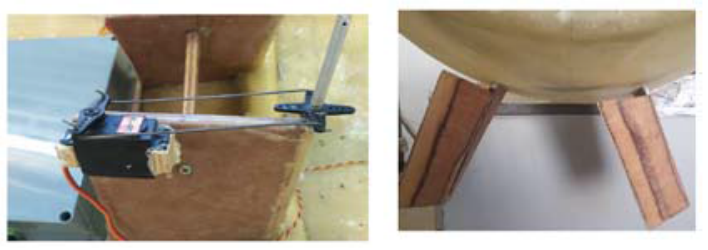

Figura 14: Servo de timón. Timones [7]

Por su parte, el servomotor del alerón de la vela es un DS-561WP de la marca Corona con un par de $10 \mathrm{~kg} \cdot \mathrm{cm}$ a $6 \mathrm{~V}$, ligero y compacto, ideal para su instalación en la vela.

\subsection{ALIMENTACIÓN}

El velero cuenta con dos placas solares y dos baterías. La placa solar principal está situada sobre la cubierta $\mathrm{y}$, a través de un circuito de carga/descarga, alimenta a la batería principal que es la encargada de energizar todos los instrumentos de navegación y medición. Para ello cuenta con dos conversores de $12 \mathrm{~V}$ a $5 \mathrm{~V}$ de 3 amperios. Uno de los conversores está dedicado exclusivamente a la Raspberry Pi la cual, a su vez, alimenta la placa Arduino por medio de la conexión USB, el LIDAR y la cámara NoIR.

La segunda placa solar está situada en el contrapeso de la vela, está conectada a una batería de litio de $3.7 \mathrm{~V}$ y su función es alimentar al procesador ESP32 responsable del servo del alerón (ver Figura 15).

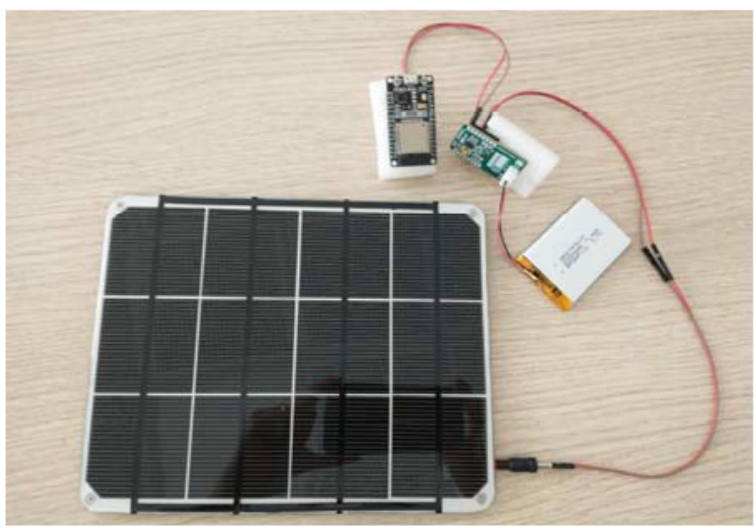

Figura 15: ESP32, placa solar y batería (alerón de la vela)

\section{RESULTADOS EXPERIMENTALES}

En las pruebas de mar realizadas el velero ha superado satisfactoriamente todas las pruebas de estanqueidad, flotabilidad y estabilidad. El sistema propulsivo ha sido capaz de aprovechar satisfactoriamente condiciones de viento moderadas (alrededor de 15 nudos) y alcanzar correctamente diversos waypoints predefinidos con una precisión razonable dadas las condiciones de viento y oleaje.

Para hacer el seguimiento en las pruebas de mar cerca de la costa, una herramienta muy útil es el Boat Track, de Boat Security, que permite trazar la ruta sobre el Google Maps a partir de las lecturas del GPS. La Figura 16 muestra la ruta de una de las primeras salidas del Sensailor en el puerto de Barcelona.

Con respecto a la maniobra se observa que por debajo de 5 nudos de viento la navegación es lenta y el pilotaje autónomo se puede ver dificultado. A fin de resolver este inconveniente, actualmente se está 
trabajando en un nuevo perfil de vela, capaz de responder mejor a bajas velocidades de viento.

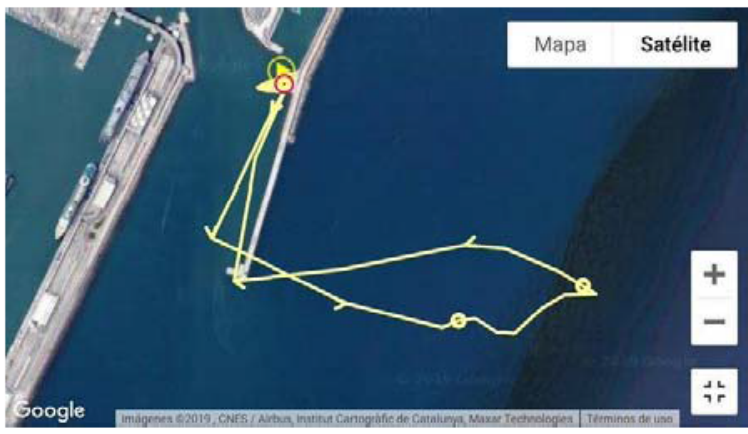

Figura 16: Seguimiento con Boat Track

Se ha comprobado que la transmisión Bluetooth entre la Raspberry $\mathrm{Pi}$ y el servo del alerón [1] es una solución efectiva al problema de torsión de los cables del interior del mástil del primer prototipo [7]. Por otro lado, se está estudiando la implementación de un mástil que permita el giro de $360^{\circ}$.

En cuanto a la adquisición y transmisión de datos, se ha comprobado que las tramas utilizadas son adecuadas y permiten registrar adecuadamente los datos de navegación y mediciones (ver Figura 17). Notar asimismo que el diseño permite la inclusión de nuevos sensores que permitirán hacer medidas de transparencia, salinidad, etc.

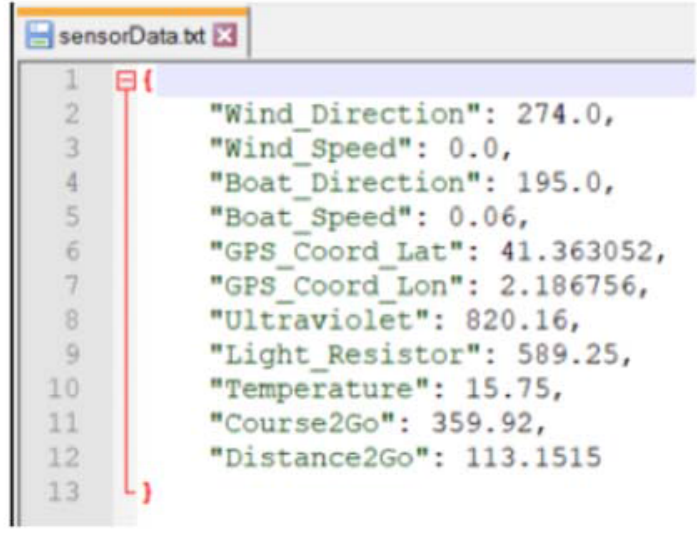

Figura 17: Lecturas de los sensores

\section{CONCLUSIONES}

Las embarcaciones autónomas de pequeña eslora son una opción económica y versátil para la recogida de datos oceanográficos. Los veleros autónomos, al ser propulsados por viento, pueden dedicar la energía captada por placas solares y almacenada en baterías a la alimentación de los sensores y actuadores. En este trabajo se ha descrito la construcción e instrumentación del velero Sensailor y la gestión de los datos por parte del sistema de control. Los resultados experimentales en pruebas de mar demuestran la viabilidad de la propuesta y apuntan a diversas líneas de mejora, especialmente en la necesidad de conseguir un giro de $360^{\circ}$ en el mástil y la posibilidad de usar velas con diferentes perfiles según las necesidades.

\section{Agradecimientos}

Los autores agradecen a la Facultat de Náutica de Barcelona la ayuda económica para la realización del proyecto Sensailor, así como a todos los patrocinadores que han colaborado aportando equipos $\mathrm{y}$ dispositivos.

\section{English summary}

\section{DEVELOPMENT AND \\ INSTRUMENTATION OF A 2 METERS LENGTH AUTONOMOUS SAILBOAT FOR THE COLLECTION OF OCEANOGRAPHIC DATA}

\begin{abstract}
This contribution presents the construction and automation of a 2 meters long sailboat designed to autonomously navigate and carry out scientific measurements. The instrumentation and the collection of information by the different sensors are described. The transmission of this information is carried out in the form of a frame to the main processor, which executes a control algorithm to generate the commands to the rudder servos and the wing aileron servo. The main processor is also responsible for presenting the data in the JavaScript Object Notation (JSON) format. Specific aspects of the maneuvering of the sail are discussed and the experimental results of the first sea trials are presented.
\end{abstract}

Keywords: Autonomous navigation, 2 meters long sailboat, Raspberry Pi, electronic instrumentation.

\section{Referencias}

[1] Chacón, E. (2021) Autonomous Sailboat Prototype Sensors and Electronics Implementation with Machine Learning for Navigation, Master Thesis, Escola Tècnica Superior d'Enginyeria de Telecomunicacions de Barcelona, Universitat Politècnica de Catalunya, Barcelona, España. 
[2] Fernández Avelino, X. (2021) Diseño $e$ implementación de un dispositivo de localización de bajo coste para la pesca de palangre: construcción de un prototipo de boya, Trabajo Final de Grado, Facultat de Nàutica de Barcelona, Universitat Politècnica de Catalunya, Barcelona, España.

[3] Kulbiej E., P. Wołejsza (2017) "Naval Artificial Intelligence", The International Conference on Marine Navigation and Safety of Sea Transportation (TRANSNAV 2017), Maritime University of Szczecin, Szczecin, Poland.

[4] Larsson, L., Eliasson, R. E. (2000) Principles of Yatch Designs. 2a ed. TAB Books Inc., Londres, UK.

[5] Liu Z., Zhang Y., Yu, X., Yuan C. (2016) "Unmanned surface vehicles: An overview of developments and challenges", Annual Reviews in Control, vol. 41.

[6] Martin B., Tarraf, D.C., Whitmore, T.C., DeWeese, J., Kenney C., Schmid J., DeLuca, P. (2019) Advancing Autonomous Systems. An Analysis of Current and Future Technology for Unmanned Maritime Vehicles, Report RAND Corporation, Santa Monica, US.

[7] Sastre, J., Manich, C., (2020) Disseny $i$ construcció d'un dron de navegació automàtica a vela. Trabajo Final de Grado, Facultat de Nàutica de Barcelona, Universitat Politècnica de Catalunya, Barcelona, España.

[8] Valios Arregui, A., (2012) Diseño de un velero de 60' para regatas oceánicas clase IMOCA 60. Proyecto Final de Carrera. Universidad de Cádiz, España.

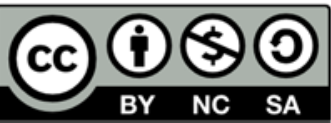

2021 by the authors. Submitted for possible open access publication under the terms and conditions of the Creative Commons Attribution CC BY-NC-SA 4.0 license (https://creativecommons.org/licenses/byncsa/4.0/deed.es). 